\title{
Editorial
}

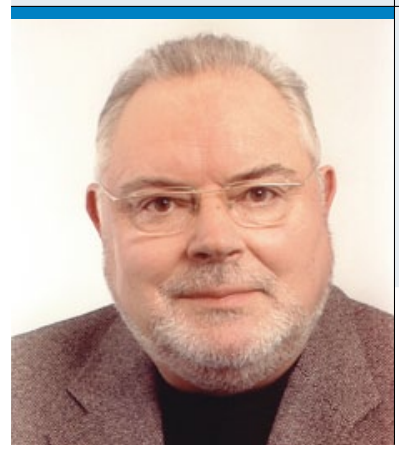

Wir HNO-Fachärzte können nach genauer Diagnostik sehr präzise entscheiden, ob eine Antibiose sinnvoll ist oder nicht.

Dr. med. Dieter Leithäuser

Chefredaktion und HNO-Arzt, Warburg

\section{Alle Jahre wieder}

W ir Ärzte werden nach alter Tradition mit den Vorwürfen überschüttet, unsere Antibiotikaverschreibungen wären maßlos überzogen und höchst unwirtschaftlich. Auch die Gruppe der HNO-Ärzte bekommt den Stempel „kleine Fachgruppe mit einem hohen Gesamtanteil aller Antibiotikaverschreibungen im Verhältnis zu anderen Fachgruppen" aufgedrückt. Das Ganze wird diesmal ausgerechnet von dem bekannten Medienunternehmen Bertelsmann publiziert - mit großem medialen Aufwand, die üblichen wenig aussagekräftigen Kreisdiagramme inklusive.

Natürlich haben wir das Umdenken gelernt und behandeln heute eine unkompliziert verlaufende Otitis media auch einmal rein lokal. Das gilt ebenso für das laufende Ohr bei liegendem Paukenröhrchen.

Den vorläufigen Höhepunkt bildet eine angeblich überzeugende Studie aus Amerika, der die Ärztezeitung am 20. Februar sogar ihre erste Seite gewidmet hatte. Patienten mit akuter Rhinosinusitis, so heißt es dort, sollten selbst bei bakterieller Ursache gemäß den Leitlinien nur in Ausnahmefällen mit Antibiotika behandelt werden - die neue Studie würde dieses Statement klar belegen. 166 Patienten mit eitrigem Nasensekret und Kiefer- oder Gesichtsschmerzen erhielten zehn Tage lang entweder täglich $1.500 \mathrm{mg}$ Amoxicillin oder Placebo. Patienten der Verumgruppe waren ihre Beschwerden nicht schneller los als Patienten der Placebogruppe. Selbst bei Risikofaktoren wie Rauchen, frühere Sinusitis oder Asthma hatte das Antibiotikum keinen Nutzen gegenüber der Placebogruppe.

Ist das für Sie überzeugend? Ich denke nicht! Wir HNO-Ärzte verfügen über die Möglichkeit, mit dem Ultraschall die spezifischen Sinusitis-Echos zu erkennen. Und das Endoskop kann uns eindeutig Eiter im mittleren Nasengang zeigen. Bei uns entscheidet der Facharzt also sehr präzise, ob eine Antibiose bei routinemäßig erkennbaren Symptomen sinnvoll erscheint. Ganz anders in Amerika: Dort bleibt der
Patient fast ausnahmslos in den Händen des Allgemeinmediziners. Nur schwerste Fälle werden notgedrungen zum Facharzt überwiesen, der gegenüber europäischen Verhältnissen auch ganz andere Schwerpunkte setzt.

Verlassen wir also nicht unsere gekonnt-gewohnten diagnostischen Pfade und entscheiden individuell richtig zum Wohle unserer Patienten. Es soll uns nicht so gehen, wie mein "Lieblingsdichter" Eugen Roth (gewissermaßen ein medizinischer Heinz Ehrhard) es in Bezug auf unser geliebtes Fach formuliert hat:

Versagen der Heilkunst
Ein Mensch, der von der Welt Gestank
seit längrer Zeit schwer nasenkrank,
der weiterhin auf beiden Ohren
das innere Gehör verloren,
und dem zum Kotzen ebenfalls
der Schwindel raushängt schon zum Hals,
begibt sich höflich und bescheiden
zum Facharzt für dergleichen Leiden.
Doch dieser meldet als Befund,
der Patient sei kerngesund,
die Störung sei nach seiner Meinung
nur subjektive Zwangserscheinung.
Der Mensch verlor auf dieses hin
den Glauben an die Medizin

(Eugen Roth, 1895-1976, ,, Rezepte vom Wunderdoktor", Carl Hanser Verlag)

Ihr
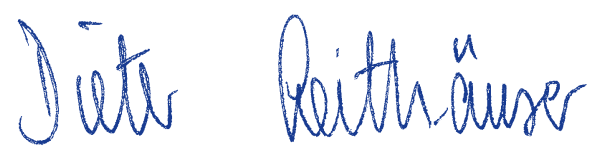